\title{
Brachytherapy practice during the COVID-19 pandemic: a review on the practice changes
}

\author{
Mansi Barthwal, MD, Vibhay Pareek, DNB, Supriya Mallick, MD, DNB, Assist. Prof., Prof. D N Sharma, MD \\ Department of Radiation Oncology, National Cancer Institute, AllMS, New Delhi, India
}

\begin{abstract}
The COVID-19 pandemic has caused a havoc across the globe, and has significantly affected oncology services, especially radiation therapy due to the need of social distancing as a measure for the pandemic mitigation. Brachytherapy, being an integral part of radiation therapy, posts a dilemma related to the practice of evidence-based oncology. It requires a significant amount of resources and personnel, thereby increasing the risk of exposure to the virus. There has been a significant amount of papers published providing the best available alternatives to external radiation; however, there is a lack of literature on the practice of brachytherapy. In times of the pandemic, deploying brachytherapy as a treatment modality can act as a double-edged sword and therefore, judicious use is warranted in such times of crisis. In this article, we provide a comprehensive review of the role of brachytherapy in various forms and different malignancy sites.

J Contemp Brachytherapy 2020; 12, 4: 393-396 DOI: https://doi.org/10.5114/jcb.2020.97643
\end{abstract}

Key words: brachytherapy, COVID-19, intracavitary brachytherapy, interstitial brachytherapy, guidelines.

\section{Purpose}

The world, as we write this review, is facing a pandemic of catastrophic proportions in the form of novel coronavirus SARS-CoV-2 (causing disease called COVID-19) [1], first identified in Wuhan, China in December 2019 [2]. Public health workers believe that the pandemic will escalate, and it will continue to last for a long time [3]. Oncological institutes, in particular, have been in the dilemma for management of patients and providing treatment options for already immunocompromised patients, as they have proven to have a five-fold relative risk for severe manifestations compared to the general population [4]. The COVID-19 pandemic infection has led to a drastic reduction of all operating room capacity, except for critical emergencies, and has posed the questions for infection prevention, rationalization of clinical workload, and working practice. Literature from various oncological institutes and societies has paved the way for a better understanding of the management of the disease. Various subsites have been explained in individual publications. We attempt to present a comprehensive review of the literature published related to the role of brachytherapy and its judicious use in the time of pandemic. In this article, various modalities of brachytherapy and individual disease sites were considered to help radiation oncologists take the best decisions for the management of oncological patients.

\section{Methodology}

We performed a PubMed search with the following MesH terms: coronavirus, COVID-19 AND oncology, coronavirus AND radiation therapy, coronavirus AND brachytherapy, along with articles published in various oncology societies through Google search and individual websites. A total of 12 articles were found to be suitable for the review. The focus was on the best available practice in terms of brachytherapy in institutes concerning medical professional working protocols, individual disease sites, and safety measures while handling the patients.

\section{Need for the review}

Brachytherapy has an established role as a treatment modality in various malignancies, providing the advantage of dose-escalation, delivering conformal treatment, and reducing toxicities associated with external beam radiation therapy. Malignant cases have a higher tendency to be infected in time of a pandemic due to compromised immune system. In times where the management decisions are driven by evidence-based procedures, employing the techniques of brachytherapy can be a challenging task due to logistics involved, providing social distancing for disease mitigation. Pandemics such as the COVID-19, call for undivided decisions to establish a collaboration between institutes, taking combined 
treatment calls, and providing brachytherapy services, where the treatment of COVID-19 patients is not taking place, or establishing protocols for maintaining utmost care while treating in centers with positive patients. The use of brachytherapy as a treatment modality has a potential for perioperative contamination and maintaining an operative workflow in times of the pandemic is a major challenge. The following review provides various sites of malignancies, in which brachytherapy can be used as a treatment modality. The precautions and measures to be taken by the healthcare personnel have been defined in several articles $[5,6,7]$ and in this review, we focus primarily on the role of brachytherapy during the time of COVID-19.

\section{Results}

\section{General principles to be followed}

Careful triage evaluation before patient hospitalization with careful questioning and clinical examination.

Polymerase chain reaction (PCR) testing of patients undergoing brachytherapy; if available in the treating institute or center, it should be strongly recommended for COVID-19 detection.

Health workers, including doctors, nursing staff, and operating room staff should be provided with the necessary protective gear.

The time in the operating/procedure room as well as brachytherapy treatment should be minimized to reduce the risk of infection.

The operating/ procedure room should have one doctor, one nurse, and one anesthetist to minimize the risk of exposure, and one physicist if real-time planning is employed.

The majority of cases should be under local or loco-regional anesthetics, and a decision of absence of anesthetists and intubation facilities need to be considered before the procedure.

If general anesthesia is required, specific anesthesiology protocol should be taken into account with dedicated protective equipment for the healthcare staff to reduce the risk of infection.

To consider brachytherapy schedules with single implant rather than having multiple implantations and minimize the length of stay in hospital.

\section{Locations and role of brachytherapy}

\section{Gynecological malignancies}

Intrauterine brachytherapy is an essential part of the treatment for cervical cancer that cannot be omitted in the curative setting.

In the resource-limited set-up, a reference to another center for brachytherapy (without severely affecting overall treatment time) is advised. When that is not feasible, external beam radiation therapy (EBRT) boost should be considered.

An experts' board should prioritize patients according to the potential benefit of brachytherapy depending on EBRT response, age, nodal involvement, and overall treatment time (OTT). A virtual tumor board via online meetings can also support best decisions.

Another alternative is to deliver 2 or 3 fractions per insertion with a gap of at least 6 hours between fractions. In the absence of spinal or general anesthesia, small diameter applicators using a local anesthetic or mild sedation can be used for brachytherapy.

Magnetic resonance imaging (MRI)-based planning may be performed at least for the first fraction, especially in bulky residual disease after EBRT requiring interstitial needles. Computed tomography (CT)-based planning is acceptable for all other cases.

Image-guided adaptive planning is preferred in gross residual disease cases at the time of brachytherapy and simple point A-based planning is sufficient for low-volume good responders.

Even after all efforts, if there is a break in treatment, extending the OTT to more than 7 weeks should be considered, adding approx. 5 Gy per week with 3D image-based brachytherapy for each week of radiation duration beyond 7 weeks, respecting organs at risk (OARs) tolerance doses [8].

Brachytherapy treatment in vulvar cancer cases should be considered as a lower priority than cervix cancer patients and should only be carried out when operation theatre capacity allows it.

Brachytherapy management according to site and stage is presented in Table $1[9,10,11,12,13,14]$.

\section{Accelerated partial breast irradiation in breast malignancies}

Accelerated partial breast irradiation (APBI) involves partial breast irradiation (tumor cavity with adequate margin), delivered in an accelerated regimen over 1-2 weeks. It has been considered as an acceptable treatment option for more than a decade.

APBI can be offered to patients with infiltrating ductal carcinoma over 60 years, tumor size up to $2 \mathrm{~cm}$, node-negative, ER+, no lymph-vascular space invasion (LVSI), and clear margins. Target volume includes the surgical cavity with a margin.

APBI can be delivered through balloon brachytherapy, interstitial brachytherapy, and external beam radiation therapy. However, in the present COVID-19 pandemic, it is advisable to apply external beam radiation therapy using three-dimensional conformal radiotherapy (3D CRT) or intensity-modulated radiation therapy/ volumetric modulated arc therapy (IMRT/VMAT), as brachytherapy would require additional resources and would lead to unnecessary exposure and further hospital stay. It may also increase the risk of transmission during intubations or upper endoscopic procedures, requiring using additional personal protective equipment (PPE).

Regimens include 30 Gy in 5 fractions every other day (Florence regimen), 38.5 Gy in 10 fractions twice daily, and $40 \mathrm{~Gy}$ in 10 daily fractions, amongst others. In the present COVID-19 pandemic, shifting to APBI for eligible patients will drastically relieve the radiation service resources. There are institutions in various countries releasing documents regarding these procedures during the pandemic [15]. 
Table 1. Brachytherapy in gynecological malignancies

Management of cervical malignancies

\begin{tabular}{|c|c|}
\hline Site of cancer & Brachytherapy \\
\hline $\begin{array}{l}\text { Stages (FIGO 2019) IB3, IIA2-IIIC2, and early IVA } \\
\text { (focal infiltration of bladder or rectum: } 1 \times 1 \mathrm{~cm} \text { ) }\end{array}$ & $\begin{array}{l}\text { Intracavitary HDR brachytherapy } 3 \text { fractions }\left(\mathrm{EQD}_{2} \text { of at least } 85 \mathrm{~Gy}\right. \\
\text { to point } \mathrm{A})[9,10] \\
\text { If theatre/anesthesia limitations present, consider EBRT boost } \\
\text { (18 Gy in } 10 \text { fractions) }\end{array}$ \\
\hline Stages IA1, IA2, IB1, IB2, IIA1 & Vault brachytherapy 6 Gy $\times 2$ fractions where indicated [11] \\
\hline Stages IVA (frank bladder or rectal infiltration) or IVB & No $[12,13]$ \\
\hline \multicolumn{2}{|c|}{ Management of endometrial malignancies in various stages } \\
\hline Site of cancer & Brachytherapy \\
\hline Stages IA, grades $1-3$ and IB, grades $1-2$ & $\begin{array}{l}\text { Vault brachytherapy if positive margins, suboptimal surgery } \\
\text { (defer if possible) }\end{array}$ \\
\hline Stage IB, grade 3 and stage $\|$ & $\begin{array}{l}\text { Vault brachytherapy: can consider only brachytherapy (no EBRT) in stage IB } \\
\text { grade } 3 \text { and stage II, grade } 1 \text { and 2, with no high-risk features }\end{array}$ \\
\hline Stages IIIA-IIIC & Vault brachytherapy [14] \\
\hline Stage IVB & No \\
\hline
\end{tabular}

Peri-operative catheter placement can help minimize the treatment duration and stay within the hospital and help deliver the required radiation dose without compromising the survival outcomes.

\section{Prostate malignancies}

In the Indian setting, prostate cancer is not a heavy burden on radiotherapy systems compared to other cancers. Moreover, its favorable prognosis and good response to androgen deprivation therapy (ADT) allows a safe delay of radical treatment for many months. This helps to reduce the overall burden on the system and staff considerably. Thus, it is possible to divert resources to more urgent needs during the COVID-19 pandemic.

Brachytherapy should be avoided as far as possible, as the availability of theatres and anesthesiologists is limited.

In centers where prostate brachytherapy is commonly performed:

- all high-dose-rate (HDR) monotherapy cases (2 implants) should be converted to HDR boost (single implant 15 Gy in 1 fraction) if the operation theatre resources permit. If not, switching to EBRT or starting of ADT should be applied,

- EBRT schedules that are due for HDR boosts (15 Gy in 1 fraction) can be converted to $37.5 \mathrm{~Gy} / 15$ fractions,

- in centers with experience and practice, brachytherapy can be delivered using low-dose-rate (LDR) brachytherapy as per isotope used.

\section{Interstitial brachytherapy}

The common sites where interstitial brachytherapy plays a role, include head and neck malignancies, soft tissue sarcoma, and anal canal malignancies. They should be avoided in time of a pandemic and planned for treatment with external beam radiation therapy. However, sites where brachytherapy can be employed as a sole treatment modality, such as the lip, oral mucosa, or penile region, should be considering as cases with priority, taking into account the superiority of this treatment modality and better cure rates [16]. As mentioned, anesthesiology protocol needs to be considered and followed with utmost care [17]. If facilities are available, intra-operative high-dose-rate brachytherapy (IO-HDR BT) can be utilized as it reduces the need for external beam radiotherapy, and the duration of hospital stay can be significantly reduced.

\section{Palliative brachytherapy}

Brachytherapy in a palliative setting should be avoided and can be replaced by hypofractionated EBRT. Other alternative and non-invasive procedures can be employed for the management of such disease states [16].

\section{Surface mold}

Adjuvant treatment in skin malignancies should be delayed until it is deemed suitable to the institute to resume a routine treatment.

If only radiotherapy (RT) to primary site is indicated, surface mold can sometimes be a preferred option, as hypofractionated radiotherapy can be delivered in a twicedaily fractionation.

\section{Pediatric malignancies}

In highly specialized centers, brachytherapy can be employed for pediatric indications, especially pediatric rhabdomyosarcoma [18].

\section{Treatment of COVID-19-positive patients}

The treatment needs to be postponed till recovery from the active COVID infection. 
If facilities are available in the hospital to handle COVID-positive patients, the treatment should be performed with respect the OTT.

After recovery, the treatment can be accelerated by delivering multiple fractions on the same day (at least 6 hours apart).

COVID-19-positive patients should not be treated with non-infected patients on the same treatment remote afterloader.

If treated:

- treatments should be performed at the end of the day,

- the machine needs to be covered with plastic sheets, which are to be changed after every treatment,

- machine and transfer tubes need to be cleaned thoroughly after each treatment,

- patients should wear a mask throughout their stay in hospital,

- single fraction delivery per day should be preferred to minimize contact with other individuals.

\section{Conclusions}

Over the next few months, the entire oncology community will see a major change in the management of strategies till the pandemic lasts, and along with the best available treatment options, counseling of the patient will also take center stage. Brachytherapy, when delivered with the utmost care, can be a useful modality during the time of the COVID-19 pandemic. In this article, we attempted to accumulate guidelines from all over the world to help clinicians achieve well-planned decisions related to radiation therapy. The guidelines are ever-evolving and structured plans depicted in the article may change with potential developments in the future.

\section{Disclosure}

The authors report no conflict of interest.

\section{References}

1. Guan W, Ni ZY, Hu Y et al. Clinical characteristics of coronavirus disease 2019 in China. N Engl J Med 2020; 382: 1708-1720.

2. Johns Hopkins University Center for Systems Science and Engineering (CSSE). Wuhan coronavirus (2019-nCoV) global cases.

3. Fauci AS, Lane HC, Redfield RR. COVID-19 - navigating the uncharted. N Engl J Med 2020; 382: 1268-1269.

4. Liang W, Guan W, Chen R et al. Cancer patients in SARS$\mathrm{CoV}-2$ infection: a nationwide analysis in China. Lancet Oncology 2020; 21: 335-337.

5. Filippi AR, Russi EG, Magrinie S et al. COVID-19 outbreak in northern Italy: first practical indications for radiotherapy departments. Int J Radiat Oncol Biol Phys 2020; 107.

6. Dinh TK, Halasz LM, Ford E et al. Radiation therapy in King County, Washington during the COVID-19 pandemic: balancing patient care, transmission mitigation and, resident training. Adv Radiat Oncol 2020.

7. Simcock R, Thomas TV, Estes C et al. COVID-19: Global radiation oncology's targeted response for pandemic preparedness. Clin Transl Radiat Oncol 2020; 22: 55-68.

8. Gay HA, Santiago R, Gil B et al. Lessons learned from hurricane Maria in Puerto Rico: practical measures to mitigate the impact of a catastrophic natural disaster on radiation oncology patients. Pract Radiat Oncol 2019; 9: 305-321.

9. Shrivastava S, Mahantshetty U, Engineer R et al. Cisplatin chemoradiotherapy vs. radiotherapy in FIGO stage IIIB squamous cell carcinoma of the uterine cervix a randomized clinical trial. JAMA Oncol 2018; 4: 506-513.

10. Vale C, Tierney JF, Stewart LA et al. Reducing uncertainties about the effects of chemoradiotherapy for cervical cancer: A systematic review and meta-analysis of individual patient data from 18 randomized trials. J Clin Oncol 2008; 26: 58025812.

11. Peters WA, Liu PY, Barrett RJ et al. Concurrent chemotherapy and pelvic radiation therapy compared with pelvic radiation therapy alone as adjuvant therapy after radical surgery in high-risk early-stage cancer of the cervix. J Clin Oncol 2000; 18: 1606-1613.

12. Mishra SK, Laskar S, Muckaden MA et al. Monthly palliative pelvic radiotherapy in advanced carcinoma of uterine cervix. J Cancer Res Ther 2005; 1: 208-212.

13. Kim DH, Lee JH, Ki YK et al. Short-course palliative radiotherapy for uterine cervical cancer. Radiat Oncol J 2013; 31: 216-221.

14. Nout RA, Smit VTHBM, Putter H et al. Vaginal brachytherapy versus pelvic external beam radiotherapy for patients with endometrial cancer of high-intermediate risk (PORTEC-2): an open-label, non-inferiority, randomised trial. Lancet 2010; 375: 816-823.

15. Al-Rashdan A, Roumeliotis $M$, Quirk $S$ et al. Adapting radiation therapy treatments for breast cancer patients during the COVID-19 pandemic: hypo-fractionation and accelerated partial breast irradiation to address World Health Organization recommendations. Adv Radiat Oncol 2020.

16. Chargari C, Deutsch E, Blanchard P et al. Brachytherapy: an overview for clinicians. CA Cancer J Clin 2019; 69: 386-401.

17. https://anaesthetists.org/Home/Resources-publications/ Anaesthetic-Management-of-Patients-During-a-COVID-19Outbreak

18. Chargari C, Haie-Meder C, Guérin F et al. Brachytherapy combined with surgery for conservative treatment of children with bladder neck and/or prostate rhabdomyosarcoma. Int J Radiat Oncol Biol Phys 2017; 98: 352-359. 